\title{
Gobierno corporativo: Mayores exigencias de los partícipes sociales
}

\author{
doi: 10.33264/rpa.202001-16 \\ Ulises Bacho Gahona \\ Facultad de Administración, UNIACC
}

\section{Resumen}

El impacto de las mayores exigencias de los partícipes sociales sobre el gobierno corporativo de la empresa es analizado en el presente artículo. Mediante una revisión de la literatura pertinente, se describe el concepto de partícipes sociales. Se contrasta el modelo de la empresa basado en el accionista con el modelo de partícipes sociales. Posteriormente se analiza el periodo pasivo y activo que dichos modelos han tenido. Se concluye con un conjunto de desafíos que el gobierno corporativo de la empresa deberá abordar para obtener y mantener una ventaja competitiva.

Palabras claves: Gobierno corporativo, participes sociales, grupos de interés, stakeholders

\begin{abstract}
The impact of the greater demands of the social partners on the corporate governance of the company is analyzed in this article. Through a review of the relevant literature, the concept of social partners is described. The shareholder-based company model is contrasted with the social stakeholder model. Subsequently, the passive and active period that these models have had is analyzed. It concludes with a set of challenges that the corporate governance of the company must address in order to obtain and maintain a competitive advantage.
\end{abstract}

Keywords: Corporate governance, social partners, interest groups, stakeholders 


\section{Introducción}

La historia ha ido mostrando que toda entidad creada por el ser humano siempre necesita ser gobernada a fin de alcanzar los objetivos que la caracterizan. En ese contexto, desde que se inició la separación de poderes entre los accionistas y la administración de una compañía, empresa u organización, aparece la necesidad de estudiar el ámbito y alcance que tiene su gobierno corporativo.

El aporte académico al campo de estudio de la gobernabilidad corporativa ha sido de variada índole, destacando literatura que permite una comprensión de los diferentes conceptos de gobierno corporativo y su composición (Baker \& Anderson, 2010; Boubaker, Nguyen, \& Nguyen, 2012; Monks \& Minow, 2011; Tarantino, 2008; Tricker, 2015). En cada artículo académico, se destaca el ámbito y alcance del gobierno corporativo, de igual manera se analizan los roles y responsabilidades de sus integrantes, con especial énfasis en el rol del presidente del directorio y el gerente general.

Una línea de trabajos de corte estadístico (Bhagat \& Bolton, 2008; Brenes, Madrigal, \& Requena, 2011; Brown \& Caylor, 2009; Krivogorsky, 2006; Manzoni \& Islam, 2009), intenta vincular el desempeño económico de las compañías con el tipo de gobierno corporativo que ellas exhiben. Los estudios, no siempre han sido concluyentes. Sin embargo, algunos de ellos ofrecen una evidencia preliminar que correlaciona positivamente el buen desempeño de gobierno corporativo con los resultados económicos financieros de la compañía.

Los aspectos legales que circunscriben a los gobiernos corporativos y en particular estudios comparativos entre países (La Porta, Lopez-de-Silanes, \& Shleifer, 1999; La Porta, Lopez-de-Silanes, Shleifer, \& Vishny, 2000; Licht, Goldschmidt, \& Schwartz, 2005; Sahin, 2014; Schneper \& Guillén, 2004), proveen una conjunto de evidencias que convergen en el respeto a principios financieros internacionales ampliamente aceptados e identifican marcos regulatorios relativamente comunes al ámbito de los gobiernos corporativos en diferentes partes del mundo.

Estudios que relevan las buenas prácticas de gobierno corporativo, caracterizan las mejores prácticas en términos de configuración de directorios, procesos de toma de decisiones, relaciones con la administración, selección de gerentes generales, materias relacionadas con las compensaciones y formas de llevar a cabo las sesiones de trabajo, entre otros (Augustine, 2012; Hilb, 2006; Page \& Spira, 2016; Subramanian, 2015).

Una línea de investigación que se ha ido levantando con el tiempo, dice relación con el gobierno corporativo y la responsabilidad social empresarial (Aluchna \& Idowu, 
2017; Idowu, Frederiksen, Mermod, \& Juul Nielsen, 2015; Manos \& Drori, 2016; Vertigans \& Idowu, 2017). Aquí, se suele utilizar el análisis comparativo para determinar los elementos distintivos que caracterizan la responsabilidad social empresarial de las empresas, según si ellas pertenecen o no a los países más desarrollados.

No obstante, lo descrito en párrafos previos, la relación entre los stakeholders, grupos de interés o partícipes sociales y el gobierno corporativo ha sido tratada como un contraste entre los supuestos que movilizan a las compañías que exhiben un comportamiento orientado preferentemente al accionista (shareholder) y aquellas cuya gestión de alta dirección considera a los partícipes sociales (stakeholders). Esta temática, merece una mayor atención que la dada hasta ahora, sobre todo en países menos desarrollados con movimientos sociales cada vez más empoderados.

En Chile, la dinámica cultural, social, tecnológica y económica ha traído grandes cambios, ellos no sólo afectan la convivencia de los ciudadanos, sino que también determinan nuevas percepciones de la relación entre los partícipes sociales y los gobiernos corporativos de las principales empresas del país. Por supuestos, los casos de colusión, la subvaloración de los intereses de la comunidad al momento de implementar nuevos proyectos productivos y las bajas penas a delitos denominados de "cuello y corbata", entre otros, no ayudan a fomentar una relación de bienestar social.

En este artículo se establece que, los actuales partícipes sociales manifiestan mayores exigencias a los gobiernos corporativos de las empresas. Así, de ser un actor pasivo los stakeholders pasan a constituirse en actores muy activos y relevantes cuando la empresa u organización proyecta su desempeño en el futuro.

Por lo anterior, las compañías en su constante búsqueda y mantención de una esquiva ventaja competitiva deberán poner mayor atención y gestionar de manera más eficiente a sus grupos de interés.

El artículo se inicia con una breve descripción del concepto de participes sociales o stakeholders. En seguida, se contrata el modelo de los accionistas (shareholders) y el modelo de los partícipes sociales (stakeholders). A continuación, se describen los periodos pasivos y activos de los partícipes sociales. Finalmente, se identifican los desafíos de las nuevas exigencias del stakeholder en el ejercicio del gobierno corporativo.

\section{Concepto de partícipes sociales (stakeholders)}

De acuerdo con Harrison, Freeman y Sá de Abreu (2015) las partes interesadas generalmente se definen como: "individuos, grupos y organizaciones que tienen 
interés en los procesos y resultados de la empresa y de quienes la empresa depende para el logro de sus objetivos (p. 859)". Con esta definición en mente, es posible identificar stakeholders que tendrán una influyente y directa relación con los procesos y resultado de la empresa. En este sentido destacan, por ejemplo, los trabajadores, accionistas, administración, sindicatos, proveedores y clientes entre otros.

No obstante, existen otros individuos, grupos y organizaciones cuyas necesidades también se verán afectados por los movimientos competitivos de las empresas. Entonces, es posible encontrar comunidades que recibirán el impacto, positivo o negativo, por la decisión de la empresa de instalar infraestructura productiva, ampliación de plantas de ensamblaje e incorporación de maquinaria pesada. En este sentido, no siempre el accionar de la empresa es acertado. Ejemplo de esto último es la fallida instalación y puesta en marcha de la planta de cerdos de Agrosuper en Freirina, provincia de Huasco en la región de Atacama Chile.

Se agrega a lo anterior, el empoderamiento de los movimientos sociales con sus respectivos intereses, muchos de ellos, muy bien organizados y articulados, destacando aquellos altamente motivados por el cuidado medio ambiental. Es importante destacar, que detectar partícipes sociales directos puede ser relativamente simple. Sin embargo, identificar y jerarquizar a los partícipes sociales indirectos no siempre es una tarea fácil. Por supuesto, se corre el riesgo de subestimar o sobrestimar el impacto que ellos podrían tener en el proceso de toma de decisiones del gobierno corporativo.

A manera de ejemplo, en Chile se logró establecer que la planta de Celulosa Arauco Constitución evacuaba desperdicios industriales que afectaban los humedales del río Cruces, en el Santuario Natural Carlos Anwandte. El delicado ecosistema, afectado por dicha acción en el año 2004, dañó seriamente la población de una especie tradicional del lugar, el cisne de cuello negro. Como era de esperar el daño reputacional de Celulosa Arauco fue muy mediático.

Ejemplos como este y otros de nivel internacional, suscita cada vez más la atención de diferentes grupos de interés. Estos stakeholders, no están dispuestos a que el crecimiento económico sea a cualquier costo o que se realice sin considerar el bienestar social actual y futuro de la comunidad.

El inusitado y mediático caso de Greta Thunberg activista medioambiental sueca es digno de estudio. Aquí, no sólo se tiene una persona que es directa y fuerte en sus planteamientos sobre el cambio climático, a una corta edad, sino que también ha logrado una gran cantidad de adeptos y seguidores en todo el mundo, incluido Chile. Claramente, se espera la aparición de más líderes apoyando causas de toda índole. La 
pregunta es: ¿están preparados los gobiernos corporativos para convivir en esos nuevos espacios relacionales?

\section{El modelo de los accionistas (shareholders) y el modelo de los partícipes sociales (stakeholders)}

Para responder la pregunta del punto anterior, quizás sea necesario que previamente se revise la repuesta a la pregunta ¿cuál es finalmente el objetivo de la empresa? Históricamente ha primado un enfoque de respuesta denominado el modelo de los accionistas (shareholders).

Este modelo, promueve la idea de que el objetivo de la empresa es maximizar el valor de la compañía, proposición que lleva existiendo ya por más de dos siglos (Baker \& Martín, 2011, p. 1). La Escuela de Chicago y su máximo representante Milton Friedman (1962) han basado fuertemente su desarrollo de la teoría económica asumiendo los supuestos de este enfoque.

En el fondo, el modelo argumenta que las empresas que emiten instrumentos de oferta pública, el bienestar o rentabilidad del accionista se genera por el mayor valor que adquiere la acción en el mercado y/o por el reparto de dividendos que se puede generar para el accionista. Esta idea no considera a los partícipes sociales como motor de la gestión de la compañía. Llevado a un extremo, el modelo ha ocasionado en más de una oportunidad problemas éticos de alta connotación mediática. Ejemplo de esto es el caso Enron (Hosseini \& Dr. Mahesh, 2016), la crisis sub-prime (Brassett \& Clarke, 2012), el caso Parmalat en Europa (Melis, 2005) y en Chile los casos de colusión de amplia cobertura.

Por supuesto, existen compañías con culturas organizacionales fuertemente enraizadas en sólidos valores corporativos, los cuales se vivencian en el día a día y que fluyen con naturalidad en las distintas instancias de gestión y que la alejan de incurrir en situaciones que atentan contra la sociedad que las sostiene. Pero, el modelo conceptual aún persiste y tiende a buscar maximizar el valor de la compañía.

En contraposición, el modelo de los partícipes sociales, promulga que el gobierno corporativo de las empresas, en su proceso de toma de decisiones, debe considerar no sólo a sus accionistas sino que también a todos aquellos que se verán afectados directa o indirectamente por las consecuencias de dichas decisiones (Jensen, 2002). Es claro observar y así lo hace notar el autor Jensen (2002), que este modelo tiene fuertes raíces en la sociología, el comportamiento organizacional, la política de los intereses especiales y por supuesto el interés personal de la gerencia (p. 9). Así, los continuos avances multidisciplinarios y los cambios culturales que acompañan la nueva sociedad establecen nuevas premisas de planeación para el gobierno 
corporativo de la organización.

Por lo anterior, han aparecido aportes que ven la importancia de incorporar al partícipe social en el ámbito y alcance de un gobierno corporativo. Por ejemplo, Demb y Neubauer (1992) plantean que: “El gobierno corporativo es el proceso mediante el cual las corporaciones responden a los derechos y deseos de las partes interesadas (p. 9)". Ellos resaltan que es la "accountability" o responsabilidad social, la que emerge de las históricas relaciones comerciales y que debe estar presente en las opciones estratégicas que evalúa el directorio de la empresa.

Una propuesta como la descrita en párrafo precedente, no es fácil de implementar, se podría caer en un inmovilismo o parálisis por tratar de considerar todos y variados intereses de la comunidad.

Ante tamaño desafío, quizás una mirada a los principios de gobierno corporativos de la Organización para la Cooperación y el Desarrollo Económicos (OECD, 2015), podría dar una guía de pensamiento para la acción. En el capítulo IV de dicho documento se establece que: "el marco de gobierno corporativo debe reconocer los derechos de las partes interesadas establecidas por ley o mediante acuerdos mutuos y fomentar la cooperación activa entre corporaciones y partes interesadas en la creación de riqueza, empleos y la sostenibilidad de empresas financieramente sólidas (p. 37)”.

En la práctica las leyes laborales regulan la relación entre empleador y colaborador, las leyes de comercio amparan el conjunto de transacciones que se dan en una economía, las regulaciones medio ambientales y de salud protegen la vida y el medio ambiente. Así, los derechos de los partícipes sociales son establecidos por ley.

Sin embargo, aquellos aspectos no regulados por ley podrían ser dejados al arbitrio de las empresas. No habría problemas, si es que las empresas sintonizan con los intereses, necesidades y valores de la sociedad de la cual forman parte. Pero, no siempre ocurre aquello. En consecuencia, las empresas de la economía moderna deberían ir pensando en considerar cual modelo seguir y por qué. Sin embargo, es claro observar, que los tiempos de cambio estarían llevando lo vientos a un modelo de gobierno corporativo más sensible a los intereses de sus stakeholders, tanto directos como indirectos.

\section{Un período pasivo del partícipe social}

Existe evidencia sustantiva que el conflicto de agencia que se da en los gobiernos corporativos, de países desarrollado tiene su origen en la atomización de la propiedad de la empresa, así el rol del Gerente General, tradicionalmente muy empoderado, puede realizar maniobras que van en contra del ánimo societarios y/o de la comunidad (Idowu \& Caliyurt, 2014; Mäntyssari, 2005; Tricker, 2015). 
Por otro lado, en países menos desarrollados el conflicto de agencia se origina en la alta concentración de la propiedad de las empresas (Tricker, 2015), dañando muchas veces al accionista minoritario. El caso Pehuenche en Chile es un ejemplo de aquello, aquí la Corte Suprema confirmó las multas aplicadas, por la Superintendencia de Valores y Seguros de la época, a dicha empresa. En efecto, se logró demostrar que el accionista minoritario Tricahue de la Empresa Eléctrica Pehuenche S.A se vio afectado seriamente sus intereses por la negociación entre Pehuenche y su empresa matriz ENDESA Chile, la que a su vez era controlada por la empresa de origen italiano ENEL. El tamaño del mercado chileno es bastantea acotado en América del Sur, sobre todo si se lo compara con mercados de mayor tamaño como lo son Argentina, Brasil y Colombia, que más que duplican el número de habitantes en comparación con el mercado chileno. Si a ello, se añade el hecho de que, el número de competidores es reducido, como por ejemplo en la industria farmacéutica de Chile, entonces se incrementa la probabilidad de colusión.

Ante dicho escenario, el rol del partícipe social o stakeholder ha sido muy limitado en el accionar del gobierno corporativo, y por un largo período histórico, se diría que ha sido un periodo pasivo para el partícipe social. Un período muy largo.

En el contexto de DAVOS 2020, Klaus Schwab (2019), fundador y actual presidente ejecutivo del Foro Económico Mundial, señaló que frente a la opción que presenta el capitalismo del accionista (shareholder capitalism) y el capitalismo de estado, como el de China (state capitalism), él recomendaría una tercera opción, el capitalismo del partícipe social (stakeholder capitalism). Su recomendación, que la ido promoviendo desde hace cincuenta años, la considera claramente como la mejor respuesta a los desafíos sociales y ambientales de hoy.

En esencia, el reto sería el tránsito a un capitalismo en el cual la empresa justifique su existencia no sólo por la búsqueda de rentabilidad económica, sino que también considere elementos que van más allá de resultados financieros y comerciales. En otras palabras, que la concepción de agregación de valor no solo sea autorreferente, sino que además incluya a un partícipe social, que al parecer es más activo y empoderado.

\section{El período activo del partícipe social}

Los resultados de corto plazo que caracterizan a la empresa competitiva a veces no dejan espacio para reflexionar acerca de los efectos sociales de, por ejemplo, instalar una nueva planta en una localidad o comuna de una región.

El partícipe social activo, percibe a las empresas como una entidad que no sólo busca el lucro, sino que rescata de ella el hecho de que es un organismo social. Por tanto, 
sensible a los intereses de su comunidad.

Desde que los consumidores adoptan la iniciativa de un consumo responsable, esto es, un consumidor que se preocupa de, entre otros, la procedencia de las materias primas, el cuidado que la empresa provee al medio ambiente y el tipo de colorantes y saborizantes que contienen los productos que consume, se evidencia un creciente empoderamiento del consumidor. Frente a lo anterior, la sociedad ha reaccionado y ha levantado normas, reglamentos y leyes que aseguren la calidad, procedencia y en general cualquier información que favorezca el proceso de toma de decisión del consumidor y/o partícipe social. En ciertas ocasiones, los grupos de interés están muy bien informados y exigen un comportamiento ético de las empresas, por ejemplo, denuncian a las empresas cuyas condiciones laborales no tiene las condiciones mínimas de trabajo, o cuando se utiliza el trabajo infantil en procesos productivos.

Adicionalmente, los partícipes sociales tienen la capacidad de organizarse, gracias a las redes sociales y las tecnologías de información y comunicación que les permiten obtener una amplia atención mediática, sobre todo cuando protesta de forma masiva.

Consecuentemente, la transición de grupo de interés pasivo a uno considerablemente más activo, sin marcha atrás, en la que nuevas necesidades comunitarias aparecen, en donde nuevas demandas sociales se levantan y en dónde el impacto de las protestas afecta directamente la gestión de la compañía, requiere que los directorios de las empresa inmersas en esa nueva realidad asuman un enfoque con clara conciencia de inclusión de sus partícipes sociales.

\section{El desafío de las nuevas exigencias del stakeholder en el gobierno corporativo}

El trabajo y rol del gobierno corporativo es cada vez más exigente. Por un lado, se ve enfrentado a un mercado más dinámico, competitivo y con entornos caracterizados por frecuentes cambios muchas veces impredecibles. Así, cambios tecnológicos relevantes no sólo pueden dejar fuera de mercado a cualquier empresa, sino que también pueden eliminar a una industria entera, sin duda el gobierno corporativo está más exigido ante el riesgo e incertidumbre de los nuevos escenarios competitivos.

Frente a un consumidor más informado, exigente, conocedor de sus derechos y empoderado al momento de seleccionar y comprar los productos disponibles en el mercado, el desempeño de la empresa se complejiza y la responsabilidad del directorio por dirigir el accionar de la empresa adquiere una nueva dimensión. En la práctica, las empresas de altos niveles de desempeño logran superar la prueba, pero 
las empresas más relevantes hace 20 años en el mundo, ya no son las que hoy lideran los rankings de desempeño.

Si a lo anterior, se agrega la incorporación de un partícipe social más activo y muy bien informado, entonces el gobierno corporativo deberá incorporar las nuevas exigencias a sus procesos de toma de decisión estratégica.

Entre las exigencias relevantes, está el hecho de que el gobierno corporativo deberá identificar, seleccionar e implementar elementos de responsabilidad social que mejor sintonicen con sus stakeholders.

En consecuencia, los procesos de análisis estratégicos de las empresas deberán mostrar especial sensibilidad a la lectura y comprensión de las demandas particulares de los diferentes grupos de interés de la empresa. Unido a lo anterior, la formulación de la estrategia, en particular la definición de sus objetivos estratégicos deberá mostrar "sensibilidad" a los requerimientos de aquellos que se verán afectados directa o indirectamente por la concepción de futuro de la compañía. Por supuesto, no siempre se podrá dar satisfacción a todas ellas, pero los mecanismos de mitigación también pueden ser relevantes en el corto plazo.

En cuanto a la implementación de la estrategia, existen ya un conjunto de normas, regulaciones y leyes que han delimitado el espacio de acción de la empresa, en materias de impacto ambiental, requerimientos sanitarios y de salud en general que determinan una forma de implementar que sea respetuosa con los intereses de la sociedad. Sin embargo, el gobierno corporativo debe estar atento no sólo por cumplir la ley sino que debería ir más allá, si quiere lograr una ventaja competitiva sostenible. Una exigencia cada vez más creciente, es la conciencia ambiental que posee la empresa en su accionar diario. Es decir, como sus procesos productivos, actividades, rutinas y procedimiento se compatibilizan con el respeto al medio ambiente y se sintonizan con los partícipes sociales.

Por supuesto, la dinámica, los espacios de trabajo y de colaboración de un particular partícipe social como son los trabajadores, colaboradores o personas que forman parte de cultura organizacional son ámbitos de acción de las directrices de un gobierno corporativo. Aquí, la visión, misión y valores corporativos dan un marco para fomentar un buen clima laboral y establecer una cultura de alto desempeño, pero para ello la empresa no solo debe ocuparse de atraer y mantener talento, sino que también ofrecer espacio para su desarrollo. Necesidad que será cada vez más creciente en estos partícipes sociales internos, por los avances tecnológicos y cambios en los estilos de vida de las nuevas generaciones. Todo un desafío para los directorios, que ya no sólo compiten por todo tipo de recursos, sino que también por el talento humano. 
Un tema no menor, es la administración de los riesgos en el trato con los diferentes grupos de interés de la empresa. Aquí todas las herramientas del "compliance" corporativo, la selección y adopción de buenas prácticas definirán formas de ser y hacer de la empresa orientada al stakeholder.

\section{Conclusión}

Como nunca la empresa había estado tan exigida por sus grupos de interés, en este artículo se ha mostrado como la concepción tradicional de gobierno corporativo se ha visto permeada por un stakeholder más empoderado y consiente de su rol en la sociedad. El paso de un partícipe social pasivo a uno más activo ha tenido un alto impacto en la sociedad. Históricamente, los grupos de interés de la empresa no eran un tema muy relevante en las tradicionales sesiones de directorio, hoy eso cambió. En la agenda del gobierno corporativo, se revisan temas asociados a los stakeholders. El análisis estratégico, la formulación de la estrategia y su implementación, requerirá considerar cada vez más temáticas asociadas a los diferentes grupos de interés de la empresa.

Pero quizás el desafío más importante será cómo transformar a la empresa con un enfoque exclusivo en los accionistas a uno que considere también a sus partícipes sociales o grupos de interés.

\section{Referencias}

Aluchna, M., \& Idowu, S. O. (2017). Responsible corporate governance: Towards sustainable and effective governance structures. Springer.

Augustine, D. (2012). Good practice in corporate governance: Transparency, trust, and performance in the microfinance industry. Business \& Society, 51(4), 659-676.

Baker, H. K., \& Anderson, R. (2010). Corporate governance: A Synthesis of theory, research, and practice. John Wiliet \& Sons.

Baker, H. K., \& Martín, G. S. (2011). Capital structure and corporate financing decisions: Theory, evidence, and practice. John Wiley \& Sons.

Bhagat, S., \& Bolton, B. (2008). Corporate governance and firm performance. Journal of Corporate Finance(14), 257-273.

Boubaker, S., Nguyen, B. D., \& Nguyen, D. K. (2012). Corporate covernance: Recent developments and new trends. Springer.

Brassett, J., \& Clarke, C. (2012). Performing the sub-prime crisis: Trauma and the financial event. International Political Sociology, 6(1), 4-20. 
Brenes, E. R., Madrigal, K., \& Requena, B. (2011). Corporate governance and family business performance. Journal of Business Research(64), 280-285.

Brown, L. D., \& Caylor, M. L. (2009). Corporate governance and firm operating performance. Review of Quantitative Finance and Accounting(32), 129-144.

Demb, A., \& Neubauer, F. f. (1992). The corporate board: Confronting the paradoxes. Long Range Planning, 25(3), 9-20.

Friedman, M. (1962). Capitalism and freedom. Chicago, Il: The University of Chicago Press.

Harrison, J. S., Freeman, R. E., \& Sá de Abreu, M. C. (2015). Stakeholder theory as an ethical approach to effective management: Applying the theory to multiple contexts. Review of Business Management, 17(55), 858-869.

Hilb, M. (2006). New corporate governance: Succesfull board management tools. Berlin.

Hosseini, S. B., \& Dr. Mahesh, R. (2016). The lesson from Enron case - Moral and managerial responsibilities. Journal of Current Research, 8(8), 37451-37460.

Idowu, S. O., \& Caliyurt, K. T. (2014). Corportae governance: An international perspective. Springer.

Idowu, S. O., Frederiksen, C. S., Mermod, A. Y., \& Juul Nielsen, M. E. (2015). Corporate social responsibility and governance: Theory and practice. Springer.

Jensen, M. C. (2002). Value maximization, stakeholder theory, and the corporate objective function. Business Ethics Quarterly, 12(2).

Krivogorsky, V. (2006). Ownership, board structure, and performance in continental Europe. The International Journal of Accounting, 41(2), 179-197.

La Porta, R., Lopez-de-Silanes, F., \& Shleifer, A. (1999). Corporate ownership around the world. The Journal of Finance, 54(2), 471-517.

La Porta, R., Lopez-de-Silanes, F., Shleifer, A., \& Vishny, R. (2000). Investor protection and corporate governance. Journal of Financial Economics(58), 3-27.

Licht, A. N., Goldschmidt, C., \& Schwartz, S. H. (2005). Culture, law, and corporate governance. International Review of Law and Economics(25), 229-255.

Manos, R., \& Drori, I. (2016). Corporate responsibility: Social action, institutions and governance. Palgrave Macmillan.

Mäntyssari, P. (2005). Comparative corporate governance: Shareholders as a rulemaker. Springer.

Manzoni, A., \& Islam, S. M. N. (2009). Performance measurement in corporate governance: DEA modelling and implications for organisational behaviour and supply chain management. Physica-Verlag. 
Melis, A. (2005). Corporate governance failures: To what extent is Parmalat a particularly Italian Case? Corporate Governance, 13(4), 478-488.

Monks, R. A. G., \& Minow, N. (2011). Corporate governance. Hoboken, NJ: John Wiley \& Sons.

OECD. (2015). G20/OECD Principles of corporate governance. OECD Publishing.

Page, M., \& Spira, L. F. (2016). Corporate governance as custodianship of the business model. Journal of Management and Governance(20), 213-228.

Sahin, K. (2014). The legitimacy of codes of corporate governance: perspectives from developed and emerging economies. Journal of Management and Governance(19), 687-708.

Schneper, W. D., \& Guillén, M. F. (2004). Stakeholder rights and corporate governance: A cross-national study of hostile takeovers. Administrative Science Quarterly, 49(2), 263-295.

Schwab, K. (2019, 05 Abril 2020). What kind of capitalism do we want? https://time.com/5742066/klaus-schwab-stakeholder-capitalism-davos/

Subramanian, G. (2015). Corporate governance 2.0: We need to return to first principles rather than meander toward "best practices". Harvard Business Review (March).

Tarantino, A. (2008). Governance, risk, and compliance handbook: Technology, finance, environmental, and international guidance and best practices. John Wiley \& Sons.

Tricker, B. (2015). Corporate governance: Principles, policies and practices. Oxford University Press.

Vertigans, S., \& Idowu, S. O. (2017). Corporate social responsibility: Academic insights and impacts. Springer.

\section{Ulises Bacho Gahona}

Master of Business Administration, State University of New York, USA. Licenciado en Ciencias en la Administración de Empresas e Ingeniero Comercial, Universidad Católica del Norte, Chile. Certificado en Gobierno Corporativo, University of Michigan y Certificado en Planificación Estratégica Avanzada, University of California at Berkeley. Académico Núcleo Temático, Facultad de Administración, UNIACC, Chile.

Email: ulises.bacho@uniacc.edu 